\title{
Crise Renal como Manifestação Inicial de Esclerose Sistêmica Sine Scleroderma $\left.{ }^{*}\right)$
}

\author{
Renal Crisis as the Initial Clinical Manifestation in \\ Systemic Sclerosis Sine Scleroderma
}

\author{
Simone Appenzeller ${ }^{(1)}$, Percival D. Sampaio-Barros ${ }^{(2)}$, Adil M. Samara ${ }^{(3)}$ e João Francisco Marques Neto ${ }^{(3)}$
}

\section{RESUMO}

A esclerose sistêmica (ES) sine scleroderma é uma doença rara, caracterizada pelo acometimento visceral (geralmente gastrintestinal, pulmonar ou vascular periférico) da ES, na ausência da característica fibrose cutânea. Os autores descrevem um caso da rara ocorrência da crise renal esclerodérmica como manifestação inicial em uma paciente com ES sine scleroderma.

Palavras-chave: esclerose sistêmica sine scleroderma, crise renal.

\section{INTRODUÇÃO}

A esclerose sistêmica (ES) é uma doença difusa do tecido conjuntivo caracterizada por fibrose progressiva da pele e proliferação da camada íntima de artérias de pequeno e médio calibres, associados ao comprometimento visceral ${ }^{(1)}$. Apresenta um amplo espectro clínico, variando de espessamento cutâneo generalizado (ES difusa) a espessamento de pele restrito às extremidades (ES limitada). A ES sine scleroderma é uma situação rara onde o característico envolvimento visceral esclerodérmico está presente antes da pele ser afetada; embora o termo tenha sido proposto por Rodnan e Fennel em 1962(2), apenas nos últimos anos suas características clínicas e demográficas foram melhor definidas. Recentemente, Poormoghim et al..$^{(3)}$, analisando o banco de dados da Universidade de Pittsburgh, observaram que $9 \%$ dos pacientes com ES sem acometimento cutâneo difuso apresentavam ES sine scleroderma, e

\section{ABSTRACT}

Systemic sclerosis sine scleroderma (ssSSc) is a rare disorder, characterised by visceral involvement (usually peripheral vascular, gastrointestinal and pulmonary) in the absence of the characteristic skin fibrosis. The authors describe in this case report the rare occurrence of sclerodermic renal crisis (SRC) as the initial clinical manifestation in a patient with ssSSc.

Keywords: systemic sclerosis sine scleroderma, renal crisis.

propuseram critérios diagnósticos. A ocorrência da crise renal esclerodérmica (CRE) como manifestação inicial da ES é muito rara, e pode ser fatal se houver demora no diagnóstico ${ }^{(4-10)}$. Os autores descrevem a ocorrência de CRE como manifestação clínica inicial numa paciente sem o característico acometimento cutâneo da ES.

\section{RELATO DO CASO}

Em janeiro de 2002, uma paciente negra, do sexo feminino, foi admitida na Enfermaria de Emergência de nossa instituição apresentando insuficiência renal aguda e hipertensão arterial maligna de três dias de duração. Ela negava sintomas clínicos significativos prévios, e referia fenômeno de Raynaud que piorava com o tempo frio (sem microulceração de polpa digital associada). Na admissão da paciente, a pressão arterial era de $180 \times 110 \mathrm{mmHg}$ e a frequência cardíaca era de 100/min, sem febre. Apresentava uma

\footnotetext{
* Trabalho realizado na Disciplina de Reumatologia do Departamento de Clínica Médica da Faculdade de Ciências Médicas da Universidade Estadual de Campinas (FCM-UNICAMP). Recebido em 06/10/03. Aprovado, após revisão, em 16/01/04.

1. Reumatologista-Assistente. FCM-UNICAMP.

2. Assistente-Doutor. FCM-UNICAMP.

3. Professor Titular. FCM-UNICAMP.
}

Endereşo para correspondência: Dr. Percival D. Sampaio-Barros. Disciplina de Reumatologia, Departamento de Clínica Médica. Faculdade de Ciências Médicas Universidade Estadual de Campinas (FCM-UNICAMP). Barão Geraldo, Campinas, SP, CEP 13081-970. E-mail: psbarros@fcm.unicamp.br 
extensa área de leucomelanodermia na face, tronco e antebraços. Não foi evidenciada artrite, bem como alterações ao exame físico pulmonar, cardíaco e abdominal. Avaliação do fundo do olho evidenciou uma retinopatia hipertensiva moderada. Os dados laboratoriais demonstraram anemia (hemoglobina $8.4 \mathrm{~g} / \mathrm{dl}$, hematócrito $24.6 \%$ ), trombocitopenia (plaquetas $38.000 / \mathrm{mm}^{3}$ ) e disfunção renal (uréia $91 \mathrm{mg} / \mathrm{dl}$, creatinina sérica $3.8 \mathrm{mg} / \mathrm{dl}$ ). Contagem de glóbulos brancos $\left(8200 / \mathrm{mm}^{3}\right)$, e enzimas hepáticas e musculares estavam nornais.

A paciente foi tratada inicialmente como hipertensão maligna, com diuréticos e drogas vasodilatadoras. Ultrasonografia abdominal revelou rins de tamanho normal, sem evidências de alteração no fluxo sangüíneo ao exame com doppler. A despeito do tratamento, ela desenvolveu oligúria com piora progressiva da função renal. Hemodiálise foi iniciada, e uma biópsia renal foi realizada; o estudo histopatológico mostrou uma importante proliferação da camada íntima das artérias interlobuladas, com deposição de fibrina nos glomérulos e nas arteríolas, sugestivo de uma doença renal crônica. Fator antinuclear (FAN) foi positivo, padrão pontilhado; anti-DNA, anti-centrômero, anti-topoisomerase I (anti-Scl 70) e anti-RNP foram negativos.

Crise renal foi diagnosticada, iniciando-se tratamento com captopril $200 \mathrm{mg} / \mathrm{dia}$; a paciente tornou-se normotensa, embora a função renal não melhorasse.

Tomografia computadorizada de alta resolução de tórax revelou extensa área de "vidro-fosco" em ambas as bases pulmonares, sugestiva de fibrose pulmonar em atividade. O teste de função pulmonar mostrou um padrão restritivo (capacidade vital forçada $=68 \%$ do esperado). $\mathrm{O}$ esofagograma contrastado mostrou refluxo gastroesofágico e hérnia hiatal, sem alteração nas ondas peristálticas; endoscopia digestiva alta mostrou uma esofagite péptica. O eletrocardiograma e o ecocardiograma com doppler foram considerados normais. Em razão das alterações pulmonares decidiu-se iniciar pulsos endovenosos mensais de ciclofosfamida. Após um ano de tratamento com ciclofosfamida e captopril, a paciente se encontrava normotensa, porém ainda em hemodiálise, e não apresentava o característico acometimento cutâneo da ES.

\section{DISCUSSÃO}

O diagnóstico de ES sine scleroderma pode ser feito quando um paciente apresenta características clínicas e histopatológicas sugestivas de ES na ausência de acometimento da pele. A partir do momento que a maioria dos clínicos tende a suspeitar do diagnóstico de ES quando da presença do espessamento da pele, a ES sine scleroderma se torna uma doença difícil de diagnosticar. A maior série de ES sine scleroderma descrita na literatura apresentava 48 pacientes, com um predomínio do acometimento vascular periférico (98\%), gastrintestinal (79\%), pulmonar (68\%) e muscular (44\%); acometimento renal não foi observado nesta série. Acometimento visceral característico da ES limitada (hipomotilidade esofágica, fibrose intersticial pulmonar, hipertensão pulmonar isolada) associado a lesões vasculares (microulceração de polpas digitais, úlceras isquêmicas digitais ou gangrena) e capilaroscopia periungueal anormal constituem as principais variáveis observadas na ES sine scleroderma; acometimento cardíaco (miocardioesclerose) e renal (crise renal esclerodérmica) são mais encontrados na ES difusa, não sendo freqüentes em pacientes com ES sine scleroderma. A ocorrência de crise renal como manifestação inicial da ES sine scleroderma é rara, sendo constituída de relatos de caso isolados ${ }^{(4-10)}$, raramente observada nas séries de ES sine scleroderma.

O acometimento renal agudo é reconhecido há muito tempo como a mais perigosa complicação da ES difusa, representando juntamente com o envolvimento pulmonar as causas de óbito mais freqüentes nestes pacientes. A CRE é definida como insuficiência renal rapidamente progressiva acompanhada ou não de hipertensão maligna de início recente durante o curso da ES, ocorrendo em 15\% a $20 \%$ dos pacientes com ES difusa, predominando nos cinco primeiros anos de doença ${ }^{(11)}$. Supõe-se que um vasoespasmo sobreposto às lesões proliferativas intimais resulte numa isquemia do aparelho justa-glomerular, aumente a secreção de renina e a formação de angiotensina II, o que posteriormente levará a um aumento da vasoconstrição renal. Fatores de risco incluem o sexo masculino, a raça negra, as contraturas de grandes articulações, o acometimento cardíaco, o uso de corticosteróides e a ES difusa rapidamente progressiva ${ }^{(11-13)}$. Antes do advento da diálise e dos inibidores da enzima conversora da angiotensina (ECA), a CRE era invariavelmente fatal. $\mathrm{O}$ prognóstico da CRE melhorou com o uso dos inibidores da ECA que, controlando os altos níveis de renina sérica, levam a uma progressiva melhora da função renal, de modo que muitas vezes a diálise pode ser suspensa. O seguimento a longo prazo dos pacientes com ES mostra que a CRE pode ser adequadamente tratada a partir do rigoroso controle da hipertensão arterial com o uso dos inibidores da ECA, e que os mesmos devem ser continuados mesmo após o início da diálise ${ }^{(14)}$. O diagnóstico precoce é importante no sentido de que o início 
imediato do tratamento pode contribuir para a manutenção da função renal.

Nesta paciente, a ausência do acometimento cutâneo característico da ES retardou o diagnóstico; embora a paciente apresentasse distúrbios de pigmentação (leucomelanodermia), como os mesmos não são patognomônicos da ES, esta não foi diagnosticada antes do início da crise renal. Após o diagnóstico da crise renal ainda na Unidade

\section{REFERÊNCIAS}

1. LeRoy EC, Black C, Fleischmajer R, et al: Scleroderma (systemic sclerosis): classification, subsets and pathogenesis. J Rheumatol 15: 202-5, 1988.

2. Rodnan GP, Fennel RH: Progressive systemic scleroderma sine scleroderma. JAMA 180: 665-70, 1962.

3. Poormoghim H, Lucas M, Fertig N, Medsger TA: Systemic sclerosis sine scleroderma. Arthritis Rheum 43: 444-51, 2000.

4. Sanders PW, Herrera GA, Ball GV: Acute renal failure without fibrotic skin changes in progressive systemic sclerosis. Nephron 48 : 121-5, 1988.

5. Gouge SF, Wilder K, Welch P, Sabnis SG, Antonovich TT: Scleroderma renal crisis prior to scleroderma. Am J Kidney Dis 14: 236-8, 1989.

6. Zwetter U, Andrassy K, Waldherr R, Ritz E: Scleroderma renal crisis as a presenting feature in the absence of skin involvement. Am J Kidney Dis 22: 53-6, 1993.

7. Gonzalez EA, Schmulbach E, Bastani B: Scleroderma renal crisis with minimal skin involvement and no serologic evidence of systemic sclerosis. Am J Kidney Dis 23: 317-9, 1994.

8. Molina JF, Anaya JM, Cabrera GE, Hoffman E, Espinoza LR: Systemic sclerosis sine scleroderma: an unusual presentation in scleroderma renal crisis. J Rheumatol 22: 557-60, 1995. de Emergência, um inibidor da ECA foi imediatamente introduzido; mas a melhora na pressão arterial não foi acompanhada pela melhora na função renal, e a paciente evoluiu com uma insuficiência renal crônica e se encontra em diálise permanente. A introdução da ciclofosfamida endovenosa deveu-se às alterações pulmonares intersticiais observadas à tomografia computadorizada de alta resolução, conforme estabelecido na literatura ${ }^{(15,16)}$.

9. Phan TG, Cass A, Gillin A, Trew P, Fertig N, Sturgess A: AntiRNA polymerase III antibodies in the diagnosis of scleroderma renal crisis sine scleroderma. J Rheumatol 26: 2489-92, 1999.

10. Canet J-J, Castañé J, Alvarez M, Nava J-M, Llibre J: Scleroderma renal crisis sine scleroderma. Nephron 90: 119-20, 2002.

11. Steen VD: Scleroderma renal crisis. Rheum Dis Clin North Am 22: 861-78, 1996.

12. Steen VD, Medsger TA Jr: Case-control study of corticosteroids and other drugs that either precipitate or protect from the development of scleroderma renal crisis. Arthritis Rheum 41: 1613-9, 1998.

13. De Marco PJ, Weisman MH, Seibold JR, Furst DE, Wong WK, Hurwitz EL, et al: Predictors and outcomes of scleroderma renal crisis: the high-dose versus low-dose D-penicillamine in early diffuse systemic sclerosis trial. Arthritis Rheum 46: 2983-9, 2002.

14. Steen VD, Medsger TA Jr: Long-term outcomes of scleroderma renal crisis. Ann Intern Med 133: 600-3, 2000.

15. Silver RM, Warrick JH, Kinsella MB, Staudt LS, Bauman MH, Strange C: Cyclophosphamide and low-dose prednisone therapy in patients with systemic sclerosis (scleroderma) with interstitial lung disease. J Rheumatol 20: 838-44, 1993.

16. White B, Moore W, Wigley FM, Xiao HQ, Wise RA: Cyclophosphamide is associated with pulmonary function and survival benefit in patients with scleroderma and alveolitis. Ann Intern Med 132: 947-54, 2000. 